\title{
Bond Markets with Stochastic Volatility
}

\section{Rafael DeSantiago * Jean-Pierre Fouque ${ }^{\dagger} \quad$ Knut Sølna $^{\ddagger}$}

September 27, 2007

\begin{abstract}
We analyze stochastic volatility effects in the context of the bond market. The short rate model is of Vasicek type and the focus of our analysis is the effect of multiple scale variations in the volatility of this model. Using a combined singular-regular perturbation approach we can identify a parsimonious representation of multiscale stochastic volatility effects. The results are illustrated with numerical simulations. We also present a framework for model calibration and look at the connection to defaultable bonds.
\end{abstract}

\section{Contents}

1 Introduction $\quad 2$

2 Pricing Bonds 3

2.1 The Term Structure Equation . . . . . . . . . . . . . . . 4

2.2 Probabilistic Representation of the Bond Price . . . . . . . . . 5

3 Affine Models 6

3.1 General Case . . . . . . . . . . . . . . . . . 6

3.2 The Vasicek Model . . . . . . . . . . . . . . . . . . . . . 7

3.3 The Yield Curve .................. 8

*IESE, Universidad de Navarra, RSantiago@iese.edu.

${ }^{\dagger}$ Department of Statistics and Applied Probability, University of California, Santa Barbara, CA 93106-3110, fouque@pstat.ucsb.edu, Work supported by NSF grant DMS-0455982.

${ }^{\ddagger}$ Department of Mathematics, University of California, Irvine CA 92697, ksolna@math.uci.edu. 
4 The Vasicek Model with Stochastic Volatility 9

4.1 The Fast Scale Volatility Factor . . . . . . . . . . . . . . 10

4.2 The Slow Scale Volatility Factor . . . . . . . . . . . . . . 11

4.3 The Model under the Risk-Neutral Measure . . . . . . . . . . 12

5 The Bond Price with Stochastic Volatility 12

5.1 The Bond Price Expansion . . . . . . . . . . . . . . . . . 13

5.2 The Fast Scale Correction . . . . . . . . . . . . . . . . . . . 14

5.3 The Slow Scale Correction . . . . . . . . . . . . . . . . 17

5.4 The Bond Price Approximation . . . . . . . . . . . . . 17

6 Group Parameter Reduction 18

6.1 Yield Correction . . . . . . . . . . . . . . . . . . 22

7 Calibration of the Model 23

8 Connection to Defaultable Bonds 24

\section{Introduction}

In this paper we illustrate the role of stochastic volatility in the case of interest rate markets. Our main example is the pricing of zero-coupon bonds when the interest rate is defined in terms of a Vasicek model, as well as the pricing of options on bonds. We use the singular perturbation framework set forth in [7] and extend the results in [4] to the case where the volatility is driven by a slow process in addition to the fast considered there.

The fact that zero-coupon bonds are parameterized by two time indices (the time at which the contract begins, and the maturity date) means that arbitrage restrictions across different maturities have to be taken into account. Also, note that options on bonds can be written on an infinite number of bonds indexed by their different maturity date and each bond cannot be treated independently, for bonds of different maturities are correlated. The most important difference between the classic Black-Scholes scenario and interest rate markets is the fact that the short rate is not the price of a traded asset.

The main building block to price many other financial instruments in interest rate markets is the zero-coupon bond. In Section 2 we show two different approaches to price zero-coupon bonds. In the following section we review briefly a class of models that have desirable properties in terms of modelling interest rate markets, namely, the affine models for the short rate. We focus then on one such model, the Vasicek model. In Section 4 we introduce stochastic volatility in the Vasicek framework by letting the volatility be driven by two stochastic processes that vary on two different time scales. In Section 5 we compute an asymptotic approximation to the bond price. This gives a parsimonious representation that is useful for calibration purposes, as presented 
in Section 7. Finally, in Section 8, we discuss the connection to the case with a defaultable bond.

\section{Pricing Bonds}

We define a zero-coupon bond with maturity $T$ as a contract, subscribed at the present time $t$, that guarantees the holder one dollar to be paid at time $T$ (with $t \leq T$ ). We begin by assuming that, under the subjective measure $\mathcal{P}$, the short rate follows the dynamics

$$
d r_{t}=\kappa\left(t, r_{t}\right) d t+\vartheta\left(t, r_{t}\right) d W_{t}
$$

where $W_{t}$ is a standard $\mathcal{P}$-Brownian motion, and we assume that $\kappa$ and $\vartheta$ are continuous with respect to $t$, and such that they satisfy the usual conditions for a strong solution. The money market account, $B_{t}$, is defined by

$$
d B_{t}=r_{t} B_{t} d t
$$

No-arbitrage pricing consists in pricing a contingent claim (the derivative) in terms of some underlying asset. In the Black-Scholes setting one typically has two processes: one that represents the price of the risky asset (usually a stock), and another one that represents the money market account.

In our case we also have two processes, $r_{t}$ and $B_{t}$, given by (1) and (2), and it would seem natural to price the zero-coupon bond as a "derivative of the short rate": that is, in order to compute the no-arbitrage price of the bond we would like to find a replicating strategy, based on the money account and some underlying asset, that gives $\$ 1$ at time $T$. The problem is that equation (1) does not represent the price of a traded asset. The only asset whose price is given exogenously is the money account, so we do not have interesting ways of forming replicating strategies (or even self-financing strategies).

We can get a better understanding of what the problem is if we try to price a zero-coupon bond. This can be done in two different ways. One is to form strategies with bonds of different maturities. But note that this is quite different than what is done in the Black-Scholes case, where a typical replicating strategy consists of holdings of the money account and of the underlying risky asset: now our portfolio will contain holdings of two different contingent claims, that is, of two derivatives (two bonds of different maturities), and maybe of the money account. If we consider the price process of one of the bonds as given, then we could price the other bond relative to the given benchmark bond.

The other way is to find an appropriate martingale measure that allows us to compute the price according to the general theory of derivative pricing. A zero-coupon bond can be considered as a contingent claim with payoff equal to one. Hence, the bond price, $P(t, T)$, is given by

$$
P(t, T)=E^{\mathcal{Q}}\left[e^{-\int_{t}^{T} r_{s} d s} \cdot 1 \mid \mathcal{F}_{t}\right]=E^{\mathcal{Q}}\left[e^{-\int_{t}^{T} r_{s} d s} \mid \mathcal{F}_{t}\right] .
$$


where $\mathcal{F}_{t}$ is the filtration associated with the Brownian motion $W_{t}$. Note however that we now need to know the equivalent martingale measure $Q$.

In the Black-Scholes case, the equivalent martingale measure was found in the following way: if we assume that the price of the underlying asset, $S_{t}$, is given by

$$
d S_{t}=\mu_{t} S_{t} d t+\sigma_{t} S_{t} d W_{t}
$$

where and $\mu_{t}$ and $\sigma_{t}$ satisfy some appropriate conditions, then the new measure $Q$ was defined by the Radon-Nikodym derivative

$$
\frac{d \mathcal{Q}}{d \mathcal{P}}:=e^{-\int_{0}^{T} \theta_{s} d W_{s}-\frac{1}{2} \int_{0}^{T} \theta_{s}^{2} d s}
$$

with $\theta_{t}=\left(\mu_{t}-r_{t}\right) / \sigma_{t}$. Note that $\theta_{t}$, and therefore $\mathcal{Q}$, are uniquely determined by the given $\mathcal{P}$-dynamics of the risky asset $\left(\mu_{t}\right.$ and $\left.\sigma_{t}\right)$.

In our case the short rate dynamics is not enough to uniquely determine the equivalent martingale measure.

\subsection{The Term Structure Equation}

In this section we recall standard arguments for obtaining the partial differential equation giving the price of a zero-coupon bond.

Assumption 2.1 We assume that the bond price depends on the short rate,

$$
P(t, T)=P\left(t, r_{t}, T\right) .
$$

We also assume, from now on, that $P(t, x, T)$ has continuous partial derivatives up to second order with respect to the first two variables, and up to first order with respect to $T$.

As we do not have a risky (underlying) asset, we form a portfolio with bonds of two different maturities. In particular, we let our portfolio contain $\theta_{1}$ bonds with maturity $T_{1}$ and $\theta_{2}$ bonds with maturity $T_{2}$. When no risk of confusion with the time indices exists we will use the notation $P_{1}=P\left(t, T_{1}\right)$ and $P_{2}=P\left(t, T_{2}\right)$ for the corresponding prices of the bonds. Applying Itô's formula we get

$$
d P_{1}=\frac{\partial P_{1}}{\partial t} d t+\frac{\partial P_{1}}{\partial x}\left(\kappa d t+\vartheta d W_{t}\right)+\frac{1}{2} \vartheta^{2} \frac{\partial^{2} P_{1}}{\partial x^{2}} d t
$$

and the analogous equation for $P_{2}$. If we let

$$
\mu_{1}=\frac{1}{P_{1}}\left(\frac{\partial P_{1}}{\partial t}+\kappa \frac{\partial P_{1}}{\partial x}+\frac{1}{2} \vartheta^{2} \frac{\partial^{2} P_{1}}{\partial x^{2}}\right) \quad \text { and } \quad \sigma_{1}=\frac{\vartheta}{P_{1}} \frac{\partial P_{1}}{\partial x}
$$

we can then write the price dynamics of the $T_{1}$-bond as

$$
d P_{1}=\mu_{1} P_{1} d t+\sigma_{1} P_{1} d W_{t} .
$$


The analogous equation holds for the $T_{2}$-bond. If we impose the condition that our strategy has to be self-financing, and we choose our portfolio in such a way that we eliminate the random part of the portfolio dynamics, then, by absence of arbitrage, this portfolio must have a rate of return equal to the short rate. This leads to a relationship between $r_{t}$ and the drift and volatility of bonds of each maturity $T$ (the details can be found in [2], Chapter 16). If we let $\mu_{T}$ and $\sigma_{T}$ have the analogous meaning as (6), but for an arbitrary maturity time $T$, then we obtain the following result.

Proposition 2.2 If the bond market is arbitrage free, there exists a process $\lambda$ such that

$$
\frac{\mu_{T}(t)-r_{t}}{\sigma_{T}(t)}=\lambda_{t}
$$

holds for all $t$, and for every maturity time $T$. The process $\lambda$ is known as the market price of risk.

Proposition 2.2 may also be expressed like this: If the bond market is free of arbitrage, bonds of all maturities must have the same market price of risk. The dynamics of the bond price, $P_{T}=P(t, T)$, is now given by

$$
d P_{T}=\mu_{T} P_{T} d t+\sigma_{T} P_{T} d W_{t}
$$

If we substitute the expressions for $\mu_{T}$ and $\sigma_{T}$ (which are given by (6) with the subscript $T$ ) in the last equation, we obtain

$$
\frac{\partial P_{T}}{\partial t}+\kappa \frac{\partial P_{T}}{\partial x}+\frac{1}{2} \vartheta^{2} \frac{\partial^{2} P_{T}}{\partial x^{2}}-r_{t} P_{T}=\vartheta \lambda \frac{\partial P_{T}}{\partial x} .
$$

As $\lambda$ is independent of maturity we don't need to keep track of $T$, so we let $P=P_{T}$. We now rearrange the last equation to obtain the following result.

Theorem 2.3 If the bond market is arbitrage free, the price of a bond of maturity $T$ is given by the boundary value problem

$$
\left\{\begin{aligned}
\frac{\partial P}{\partial t}+(\kappa-\lambda \vartheta) \frac{\partial P}{\partial x}+\frac{1}{2} \vartheta^{2} \frac{\partial^{2} P}{\partial x^{2}}-x P & =0 \\
P(T, x, T) & =1 .
\end{aligned}\right.
$$

This partial differential equation is referred to as the term structure equation.

Note that now $\lambda$ is not determined within the model: it is determined by the market.

\subsection{Probabilistic Representation of the Bond Price}

We have seen earlier that the bond price could be computed as a conditional expectation, but we did not know with respect to which measure. Proposition 2.2 shows how to construct the measure. 
Let $P^{\star}(t, T):=e^{-\int_{0}^{t} r_{s} d s} P(t, T)$. An equivalent martingale measure $\mathcal{Q}$ is said to be an equivalent martingale measure for the bond market if $Q$ is equivalent to $\mathcal{P}$ and the process $P^{\star}(t, T)$ is a martingale under $\mathcal{Q}$, for all maturities $T$. Thus, we let $\bar{T}$ denote the largest maturity and identify the measure $\mathcal{Q}$ by the Radon-Nikodym derivative

$$
\frac{d \mathscr{Q}}{d \mathcal{P}}:=e^{-\int_{0}^{\bar{T}} \lambda_{s} d W_{s}-\frac{1}{2} \int_{0}^{\bar{T}} \lambda_{s}^{2} d s},
$$

where $\lambda_{t}$ is given by (8). Then, restricted to $\mathcal{F}_{T}$ we have

$$
\frac{d \mathscr{Q}}{d \mathcal{P}}:=e^{-\int_{0}^{T} \lambda_{s} d W_{s}-\frac{1}{2} \int_{0}^{T} \lambda_{s}^{2} d s} .
$$

Proposition 2.2 guarantees the existence of the quantity $\lambda$, as determined by the market. Besides the condition imposed by Proposition 2.2, we assume that $\lambda$ is such that the process defined by

$$
Z_{t}:=e^{-\int_{0}^{t} \lambda_{s} d W_{s}-\frac{1}{2} \int_{0}^{t} \lambda_{s}^{2} d s}
$$

is a $\mathcal{P}$-martingale. In practice, $\lambda$ is frequently taken to be a constant.

If $\lambda$ is such that (11) is a martingale, by Girsanov's theorem it follows that $\tilde{W}_{t}=W_{t}+\int_{0}^{t} \lambda_{s} d s$ is a standard Q-Brownian motion. Under the new measure $Q$, the dynamics of the short rate is given by

$$
d r_{t}=(\kappa-\lambda \vartheta) d t+\vartheta d \tilde{W}_{t} .
$$

\section{Affine Models}

\subsection{General Case}

In the literature there are many different models for the dynamics of the short rate. The most popular ones are the so-called affine models, due to their pleasing properties from analytical and computational points of view. These models are characterized by the assumption that the short rate is an affine function of a vector of unobserved state variables $v_{t}=\left(v_{1}(t), \ldots, v_{N}(t)\right)$. Specifically, it is assumed that

$$
r_{t}=\delta_{0}+\sum_{i=1}^{N} \delta_{i} v_{i}(t),
$$

where the vector $v_{t}$ follows an "affine diffusion,"

$$
d v_{t}=\alpha\left(v_{t}\right) d t+\beta\left(v_{t}\right) d W_{t} .
$$

With the proper choice of $\alpha$ and $\beta$, corresponding to the affine family, one obtains that the price at time $t$ of a zero-coupon bond with maturity $T$ can be written as

$$
P(t, T)=e^{A(T-t)-B(T-t)^{\top} v_{t}},
$$


where $A$ and $B$ are obtained as solutions of a set of ordinary differential equations. The important point is that the yield curve of these models is affine in the state variables, where we define the yield curve in (24) below.

\subsection{The Vasicek Model}

Among the affine class, one of the most popular ways of modelling the short rate is the Vasicek model, in which the short rate is considered to be a Gaussian process that satisfies the following stochastic differential equation

$$
d r_{t}=a\left(r_{\infty}-r_{t}\right) d t+\sigma d W_{t}
$$

where $a, r_{\infty}$ and $\sigma$ are constants, and $W_{t}$ is a standard Brownian motion. In this case, the only state variable is the "unobserved" short rate. The drawback of this model is that, due to the Gaussian nature of the $r_{t}$ process, there is a positive probability that the short rate is negative, which is unreasonable from an economic point of view. Despite this drawback, the Vasicek model is frequently used because it allows explicit computations and many results can be obtained in closed form, making it easier to highlight the important points of further analysis.

Our first goal is to price zero-coupon bonds. Following the analysis of the previous section, we let $\mathcal{Q}$ be defined by

$$
\frac{d \mathcal{Q}}{d \mathcal{P}}:=e^{-\lambda d W_{T}-\frac{1}{2} \lambda^{2} T},
$$

where we assume the market price of risk, $\lambda$, to be a constant. Under this measure, the dynamics of the short rate is given by the Ornstein-Uhlenbeck process

$$
d r_{t}=a\left(r^{\star}-r_{t}\right) d t+\sigma d \tilde{W}_{t},
$$

where $r^{\star}=r_{\infty}-\frac{\lambda \sigma}{a}$, and $\tilde{W}_{t}=W_{t}+\lambda t$ is a standard Q-Brownian motion.

Let $\mathcal{B}_{V}\left(t, x ; T, \sigma, r^{\star}\right)$ denote the Vasicek price at time $t$ of a zero-coupon bond with maturity $T$ when $r_{t}=x$. By Theorem 2.3, this no-arbitrage price is determined by the term structure equation:

$$
\left\{\begin{aligned}
\mathcal{L}_{V}\left(\sigma, r^{\star}\right) \mathcal{B}_{V} & =0 \\
\mathcal{B}_{V}\left(T, x ; T, \sigma, r^{\star}\right) & =1
\end{aligned}\right.
$$

where the Vasicek operator for the parameters $\sigma$ and $r^{\star}$ is given by

$$
\mathcal{L}_{V}\left(\sigma, r^{\star}\right):=\frac{\partial}{\partial t}+\frac{1}{2} \sigma^{2} \frac{\partial^{2}}{\partial x^{2}}+a\left(r^{\star}-x\right) \frac{\partial}{\partial x}-x \cdot .
$$

Note that this operator depends on $\lambda$ through $r^{\star}=r_{\infty}-\frac{\lambda \sigma}{a}$. Let $\tau=T-t$ be the time to maturity. Trying a solution of the form

$$
\mathcal{B}_{V}\left(t, x ; T, \sigma, r^{\star}\right)=A(\tau) e^{-B(\tau) x},
$$


we get that $A$ and $B$ satisfy the following ordinary differential equations

$$
\begin{aligned}
\frac{\dot{A}}{A} & =\frac{1}{2} \sigma^{2} B^{2}-a r^{\star} B, \\
\dot{B} & =1-a B,
\end{aligned}
$$

with initial conditions $A(0)=1$, and $B(0)=0$ and where the dot means differentiation with respect to $\tau$ Solving these differential equations we get that

$$
B(\tau)=\frac{1}{a}\left(1-e^{-a \tau}\right)
$$

and

$$
A(\tau)=e^{-R_{\infty} \tau+R_{\infty} \frac{1}{a}\left(1-e^{-a \tau}\right)-\frac{\sigma^{2}}{4 a^{3}}\left(1-e^{-a \tau}\right)^{2}},
$$

where

$$
R_{\infty}=r^{\star}-\frac{\sigma^{2}}{2 a^{2}} .
$$

Hence, the Vasicek bond price is given by

$$
\mathcal{B}_{V}\left(t, r_{t} ; T, \sigma, r^{\star}\right)=e^{-\left\{R_{\infty} \tau+\left(r_{t}-R_{\infty}\right) B+\frac{\sigma^{2}}{4 a} B^{2}\right\}} .
$$

For fixed $t$, the graph of $P(t, T)$ as a function of $T$ is called the bond price curve at time $t$.

\subsection{The Yield Curve}

If at time $t$ we buy a zero-coupon bond with maturity $T$, the continuously compounded return on this investment, which we denote $R(t, T)$, is obtained from

$$
P(t, T) e^{(T-t) R}=1 .
$$

This quantity $R$, which gives us the "internal rate of return" of the bond, is called the yield and it plays an important role in interest rate markets. At time $t$ one would be indifferent to buy the $T$-bond or to invest the amount $P(t, T)$ during the period $[t, T]$ at the rate $R(t, T)$. If we keep $t$ fixed and we let maturity vary, we obtain very useful information about the interest rate market, namely, we get an idea of what the market thinks about the future evolution of interest rates.

The continuously compounded zero-coupon yield, $R(t, T)$, is given by

$$
R(t, T)=-\frac{\log P(t, T)}{T-t} .
$$

For fixed $t$, the graph of $R(t, T)$ is called the yield curve, or the term structure at $t$. 

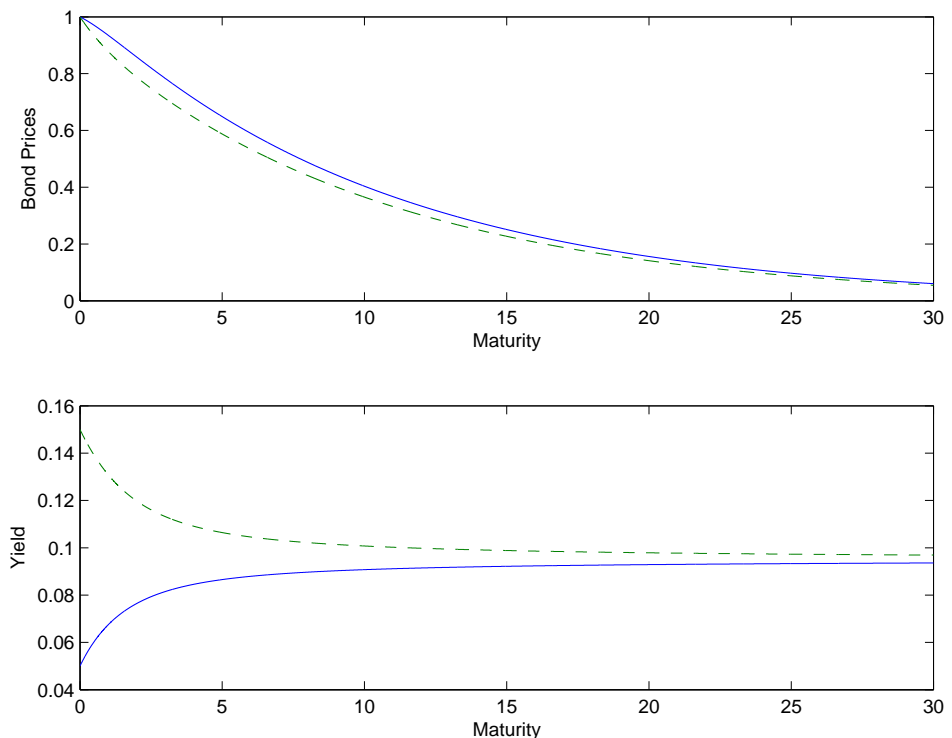

Figure 1: Bond price (top) and yield curve (bottom) for the Vasicek model. For this example, $a=1, \sigma=0.1, r^{\star}=0.1, R_{\infty}=0.095$. The initial value of the short rate is $x=0.05$ for the solid line, and $x=0.15$ for the dashed line.

The yield for the Vasicek model is given by

$$
R_{V}(t, T)=R_{\infty}+\left(r_{t}-R_{\infty}\right) \frac{B}{T-t}+\frac{\sigma^{2}}{4 a} \frac{B^{2}}{T-t}
$$

which is an affine function of the short rate $r_{t}$. In Figure 1 we show the Vasicek bond price (as a function of maturity) and the corresponding yield curve for some specific values of the parameters. We can see how the mean reverting property of the Ornstein-Uhlenbeck process brings the yield back to its long term value, $R_{\infty}$.

\section{The Vasicek Model with Stochastic Volatil- ity}

In the case of the Black-Scholes model, typical historical data of the standard deviation of returns indicate that the volatility is not constant. The distributions of returns are not normal (they show fat tails), moreover, one can observe a smile effect in the implied volatility and similarly for the bond market $[1,3]$.

Figure 2 shows two different paths of a non-constant volatility. In the top figure the volatility is low (under 14\%) for the first 17 years, and then it is high for the rest of the time. In the bottom figure the volatility is high for several months, and then low for a similar period. Then high again, and so forth. The second path exhibits volatility clustering, the tendency of volatility to come in 

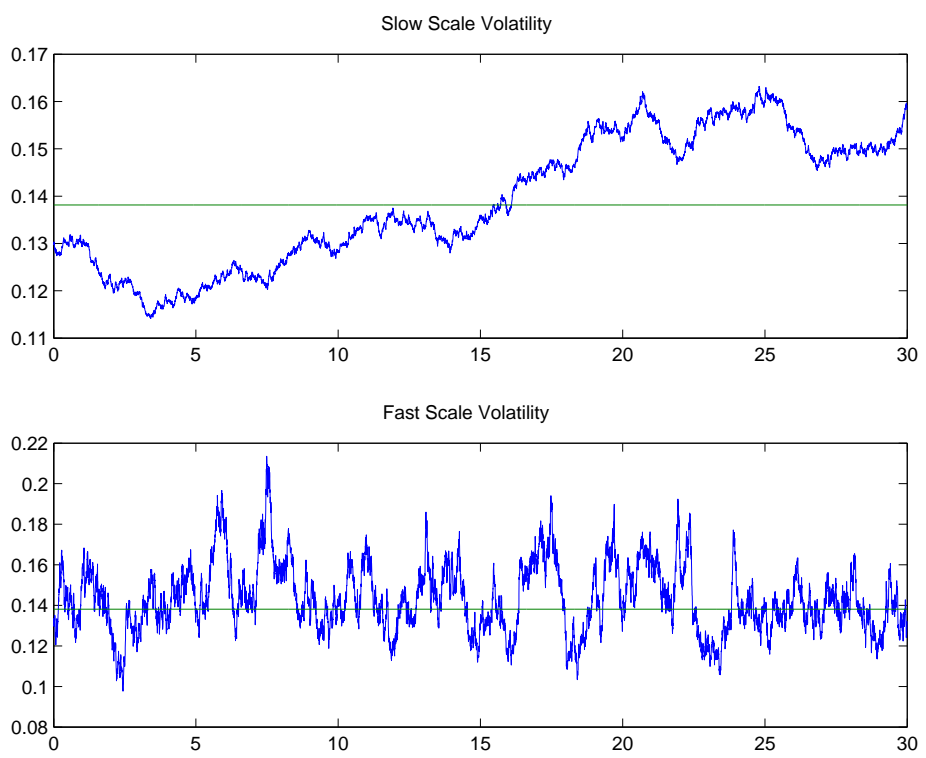

Figure 2: Volatility Paths. Top: Slow scale. Bottom: Fast scale.

rapid bursts. This burstiness is closely related to mean reversion. We want to incorporate these type of volatility variations schematically in our modeling, the analysis of such an extended model will inform us about the significance of volatility heterogeneity and the shortcoming of the constant parameter model. As we show below we can do so in a robust way, essentially, the presence of such volatility time scales is what is important, not their detailed modelling.

We now introduce a stochastic volatility model as follows. Let

$$
\sigma_{t}:=f\left(Y_{t}, Z_{t}\right)
$$

where $f$ is a smooth bounded positive function, bounded away from zero, and $Y_{t}$ and $Z_{t}$ are two diffusion processes that vary, respectively, on a fast time scale and on a slow time scale. Under the subjective probability measure $\mathcal{P}$, the short rate follows the stochastic differential equation

$$
d r_{t}=a\left(r_{\infty}-r_{t}\right) d t+\sigma_{t} d W_{t}^{0}
$$

where $a$ and $r_{\infty}$ are constants, and $W_{t}^{0}$ is a standard Brownian motion.

\subsection{The Fast Scale Volatility Factor}

We choose the first factor driving the volatility, $Y_{t}$, to be a fast mean reverting Ornstein-Uhlenbeck process

$$
d Y_{t}=\alpha\left(m-Y_{t}\right) d t+\beta d W_{t}^{1}
$$


where $\alpha, m$ and $\beta$ are constants, and $W_{t}^{1}$ is a standard Brownian motion whose covariation with $W_{t}^{0}$ is given by

$$
d\left[W^{0}, W^{1}\right]_{t}=\rho_{1} d t .
$$

We assume $\rho_{1}$ to be constant and $\left|\rho_{1}\right|<1$. The process $\left\{Y_{t}\right\}_{t>0}$ is an ergodic process whose invariant distribution is $\mathcal{N}\left(m, \nu^{2}\right)$, with $\nu^{2}=\beta^{2} / 2 \alpha$. Under the invariant distribution, the covariance is given by

$$
E\left[\left(Y_{t}-m\right)\left(Y_{s}-m\right)\right]=\nu^{2} e^{-\alpha|t-s|},
$$

which shows that the exponential rate of decorrelation of $\left\{Y_{t}\right\}$ is $\alpha$. Hence, $1 / \alpha$ can be thought of as the typical correlation time. The parameter $\nu^{2}$ controls the size of the fluctuations in the volatility associated with variations in $Y_{t}$. We assume that $\nu^{2}$ is constant and consider a regime of fast mean reversion or $\alpha$ large (i.e., $\beta=\mathcal{O}(\sqrt{\alpha})$ ). Increasing $\alpha$ and keeping $\nu$ fixed changes the degree of burstiness of the volatility without affecting the magnitude of the fluctuations. (See [7] for more details).

Define $\epsilon=1 / \alpha$. Then, the small parameter $\epsilon$ can be interpreted as the mean reversion time of the volatility associated with fluctuations in $Y_{t}$. The asymptotic analysis that we introduce in Section 5.2 is then for the case $\epsilon \downarrow 0$, with $\nu^{2}$ fixed, that is $\beta=\nu \sqrt{2 / \epsilon}$.

\subsection{The Slow Scale Volatility Factor}

We choose the second factor, $Z_{t}$, to follow the stochastic differential equation

$$
d Z_{t}=\delta c\left(Z_{t}\right) d t+\sqrt{\delta} g\left(Z_{t}\right) d W_{t}^{2}
$$

where $\delta$ is a small parameter, $W_{t}^{2}$ is a standard Brownian motion, and we assume that the functions $c(\cdot)$ and $g(\cdot)$ are smooth and at most linearly growing at infinity. As the parameter $\delta$ is assumed to be small, this makes $Z_{t}$ vary on a slow scale: namely, $Z_{t}$ varies on the $\mathcal{O}(1 / \delta)$ scale. Note that we now have three relevant time scales:

(1) The $\mathcal{O}(1)$ scale, which is the time-to-maturity scale $(\mathrm{T})$.

(2) The slow-scale or $\mathcal{O}(1 / \delta)$ scale, which is the characteristic time scale of the process $Z_{t} .\left(T<\frac{1}{\delta}\right)$

(3) The fast-scale or $\mathcal{O}(\epsilon)$ scale, which is the mean reversion time of the process $Y_{t} .(\epsilon<T)$ 


\subsection{The Model under the Risk-Neutral Measure}

The introduction of two new sources of randomness gives rise to a family of equivalent martingale measures that will be parameterized by the market price of risk, $\lambda$, and two market prices of volatility risk, which we denote by $\gamma$ and $\xi$, associated respectively with $Y_{t}$ and $Z_{t}$. All these market prices are not determined within the model, but are fixed exogenously by the market.

We now assume that these market prices of risk do not depend on the short rate: that is, they have the form $\lambda\left(Y_{t}, Z_{t}\right), \gamma\left(Y_{t}, Z_{t}\right)$ and $\xi\left(Y_{t}, Z_{t}\right)$. We also assume that $\lambda, \gamma$ and $\xi$ are smooth bounded functions.

We define the new equivalent martingale measure, $Q$, by

$$
\frac{d \mathscr{Q}}{d \mathcal{P}}=e^{-\int_{0}^{T} H_{t} \cdot d B_{t}-\frac{1}{2} \int_{0}^{T}\left\|H_{t}\right\|^{2} d t},
$$

where $H_{t}=\left(\lambda\left(Y_{t}, Z_{t}\right), \gamma\left(Y_{t}, Z_{t}\right), \xi\left(Y_{t}, Z_{t}\right)\right)$ and $B_{t}=\left(B_{t}^{0}, B_{t}^{1}, B_{t}^{2}\right)$. By Girsanov's Theorem, $B_{t}^{\star}=B_{t}+\int_{0}^{t} H_{s} d s$ is a Q-Brownian motion, with $B_{t}^{\star}=$ $\left(B_{t}^{0 *}, B_{t}^{1 \star}, B_{t}^{2 \star}\right)$. We now define $\left(W_{t}^{0 *}, W_{t}^{1 \star}, W_{t}^{2 \star}\right)$ with the following correlation structure:

$$
\left(\begin{array}{l}
W_{t}^{0 *} \\
W_{t}^{1 \star} \\
W_{t}^{2 \star}
\end{array}\right)=\left(\begin{array}{ccc}
1 & 0 & 0 \\
\rho_{1} & \sqrt{1-\rho_{1}^{2}} & 0 \\
\rho_{2} & \tilde{\rho}_{12} & \sqrt{1-\rho_{2}^{2}-\tilde{\rho}_{12}^{2}}
\end{array}\right)\left(\begin{array}{c}
B_{t}^{0 *} \\
B_{t}^{1 \star} \\
B_{t}^{2 \star}
\end{array}\right),
$$

where we assume $\left|\rho_{1}\right|<1$ and $\rho_{2}^{2}+\tilde{\rho}_{12}^{2}<1$. Note that with this structure $d\left[W^{1}, W^{2}\right]_{t}=\rho_{12} d t$, with $\rho_{12}:=\rho_{1} \rho_{2}+\tilde{\rho}_{12} \sqrt{1-\rho_{1}^{2}}$. Under the Risk-Neutral Measure, our model is therefore:

$$
\begin{aligned}
d r_{t} & =\left(a\left(r_{\infty}-r_{t}\right)-\lambda_{t} f_{t}\right) d t+f_{t} d W_{t}^{0 *} \\
d Y_{t} & =\left(\frac{1}{\epsilon}\left(m-Y_{t}\right)-\frac{1}{\sqrt{\epsilon}} \Lambda_{t}\right) d t+\frac{1}{\sqrt{\epsilon}} d W_{t}^{1 \star} \\
d Z_{t} & =\left(\delta c\left(Z_{t}\right)-\sqrt{\delta} g\left(Z_{t}\right) \Gamma_{t}\right) d t+\sqrt{\delta} g\left(Z_{t}\right) d W_{t}^{2 \star},
\end{aligned}
$$

where we have used $\lambda_{t}$ for $\lambda\left(Y_{t}, Z_{t}\right)$, and the analogous convention for $\gamma_{t}, \xi_{t}$, $f_{t}, \Lambda_{t}$ and $\Gamma_{t}$, together with:

$$
\begin{aligned}
\Lambda(y, z) & :=\rho_{1} \lambda(y, z)+\gamma(y, z) \sqrt{1-\rho_{1}^{2}} \\
\Gamma(y, z) & :=\rho_{2} \lambda(y, z)+\tilde{\rho}_{12} \gamma(y, z)+\xi(y, z) \sqrt{1-\rho_{2}^{2}-\tilde{\rho}_{12}^{2}} .
\end{aligned}
$$

\section{The Bond Price with Stochastic Volatility}

In this section we present the asymptotic analysis for the bond price, and we find an approximation to the price that accounts for stochastic volatility. In 
order to emphasize the dependence of the approximation on the small parameters $\epsilon$ and $\delta$, we will denote the no-arbitrage Vasicek price of a zero-coupon bond with maturity $T$ by $P^{\epsilon, \delta}(t, x, y, z ; T)$. Using the probabilistic representation, this price is given by

$$
P^{\epsilon, \delta}(t, x, y, z ; T)=E^{\mathcal{Q}}\left[e^{-\int_{t}^{T} r_{s} d s} \mid r_{t}=x, Y_{t}=y, Z_{t}=z\right] .
$$

Because in the Vasicek model $r_{t}$ can take any negative value with positive probability, the expectation in (35) is not trivially finite. One can prove that the expectation is indeed finite by showing that $r_{t}$ and $\int_{0}^{t} r_{s} d s$ have exponential moments, see $[4,6]$.

\subsection{The Bond Price Expansion}

An application of Feynman-Kac's result to (35) shows that $P^{\epsilon, \delta}$ is a solution of the following problem

$$
\left\{\begin{array}{r}
\mathcal{L}^{\epsilon, \delta} P^{\epsilon, \delta}=0 \\
P^{\epsilon, \delta}(T, x, y, z ; T)=1
\end{array}\right.
$$

where the operator $\mathcal{L}^{\epsilon, \delta}$ is given by

$$
\mathcal{L}^{\epsilon, \delta}=\frac{1}{\epsilon} \mathcal{L}_{0}+\frac{1}{\sqrt{\epsilon}} \mathcal{L}_{1}+\mathcal{L}_{2}+\sqrt{\delta} \mathcal{M}_{1}+\delta \mathcal{M}_{2}+\sqrt{\frac{\delta}{\epsilon}} \mathcal{M}_{3},
$$

with

$$
\begin{aligned}
\mathcal{L}_{0} & =(m-y) \frac{\partial}{\partial y}+\nu^{2} \frac{\partial^{2}}{\partial y^{2}} \\
\mathcal{L}_{1} & =\nu \sqrt{2}\left(\rho_{1} f(y, z) \frac{\partial^{2}}{\partial x \partial y}-\Lambda(y, z) \frac{\partial}{\partial y}\right) \\
\mathcal{L}_{2} & =\frac{\partial}{\partial t}+\frac{1}{2} f^{2}(y, z) \frac{\partial^{2}}{\partial x^{2}}+\left(a\left(r_{\infty}-x\right)-\lambda(y, z) f(y, z)\right) \frac{\partial}{\partial x}-x \\
\mathcal{M}_{1} & =\rho_{2} f(y, z) g(z) \frac{\partial^{2}}{\partial x \partial z}-g(z) \Gamma(y, z) \frac{\partial}{\partial z} \\
\mathcal{M}_{2} & =c(z) \frac{\partial}{\partial z}+\frac{1}{2} g^{2}(z) \frac{\partial^{2}}{\partial z^{2}} \\
\mathcal{M}_{3} & =\nu \sqrt{2} \rho_{12} g(z) \frac{\partial^{2}}{\partial y \partial z}
\end{aligned}
$$

Note that if we fix $y$ and $z$, and we let $\sigma=f(y, z)$ and $r^{\star}=r_{\infty}-\frac{\lambda(y, z) f(y, z)}{a}$, then we can write (40) as

$$
\mathcal{L}_{2}=\frac{\partial}{\partial t}+\frac{1}{2} \sigma^{2} \frac{\partial^{2}}{\partial x^{2}}+a\left(r^{\star}-x\right) \frac{\partial}{\partial x}-x \cdot
$$


which is the Vasicek operator defined in (16). That is, $\mathcal{L}_{2} \equiv \mathcal{L}_{V}\left(\sigma, r^{\star}\right)$.

The small parameter $\epsilon$ gives rise to a singular perturbation problem. In the limit when $\epsilon$ goes to zero, the leading problem becomes the Poisson equation associated with the operator $\mathcal{L}_{0}$ rather than the Vasicek problem. The terms associated only with the small parameter $\delta$ give rise to a regular perturbation problem about the Vasicek operator $\mathcal{L}_{2}$. In the next sections we carry out the combined regular and singular perturbation expansion.

In order to carry out the asymptotic analysis, we begin writing $P^{\epsilon, \delta}$ in powers of $\sqrt{\delta}$ :

$$
P^{\epsilon, \delta}=P_{0}^{\epsilon}+\sqrt{\delta} P_{1}^{\epsilon}+\delta P_{2}^{\epsilon}+\cdots .
$$

Substituting (44) in the PDE (36), and considering the $\mathcal{O}(1)$ terms (with respect to $\delta$ ) and the $\mathcal{O}(\sqrt{\delta})$ terms, we define the problems that will determine $P_{0}^{\epsilon}$ and $P_{1}^{\epsilon}$.

Definition 5.1 The leading order term $P_{0}^{\epsilon}$ is defined as the unique solution to

$$
\begin{aligned}
\left(\frac{1}{\epsilon} \mathcal{L}_{0}+\frac{1}{\sqrt{\epsilon}} \mathcal{L}_{1}+\mathcal{L}_{2}\right) P_{0}^{\epsilon} & =0 \\
P_{0}^{\epsilon}(T, x, y, z ; T) & =1,
\end{aligned}
$$

Definition 5.2 The term $P_{1}^{\epsilon}$ is defined as the unique solution to the problem

$$
\begin{aligned}
\left(\frac{1}{\epsilon} \mathcal{L}_{0}+\frac{1}{\sqrt{\epsilon}} \mathcal{L}_{1}+\mathcal{L}_{2}\right) P_{1}^{\epsilon} & =-\left(\mathcal{M}_{1}+\frac{1}{\sqrt{\epsilon}} \mathcal{M}_{3}\right) P_{0}^{\epsilon} \\
P_{1}^{\epsilon}(T, x, y, z ; T) & =0 .
\end{aligned}
$$

In the next sections we expand $P_{0}^{\epsilon}$ and $P_{1}^{\epsilon}$ in powers of $\sqrt{\epsilon}$ to obtain the approximation to the price, $P^{\epsilon, \delta}$.

\subsection{The Fast Scale Correction}

First we expand $P_{0}^{\epsilon}$ as

$$
P_{0}^{\epsilon}=P_{0}+\sqrt{\epsilon} P_{1,0}+\epsilon P_{2,0}+\epsilon^{3 / 2} P_{3,0}+\cdots .
$$

In order to find explicit expressions for $P_{0}$ and $P_{1,0}$, we insert (49) into equation (45). From the $\mathcal{O}(1 / \epsilon)$ and $\mathcal{O}(1 / \sqrt{\epsilon})$ terms we obtain that $P_{0}$ and $P_{1,0}$ do not depend on $y$ : hence, we can write $\left.P_{0}=P_{0}(t, x, z)\right)$, and $P_{1,0}=P_{1,0}(t, x, z)$. The $\mathcal{O}(1)$ terms give that $P_{0}$ is determined by the problem:

$$
\left\{\begin{array}{r}
\left\langle\mathcal{L}_{V}\right\rangle P_{0}=0 \\
P_{0}(T, x, z)=1
\end{array}\right.
$$


where the bracket notation $\langle\cdot\rangle$ means integration with respect to the invariant distribution of the $Y$ process (ie., integration with respect to a normal $\left.\mathcal{N}\left(m, \nu^{2}\right)\right)$. That is, $\left\langle\mathcal{L}_{V}\right\rangle$ is the Vasicek operator $\mathcal{L}_{V}\left(\bar{\sigma}, \bar{r}^{\star}\right)$, with parameters

$$
\bar{\sigma}(z):=\sqrt{\left\langle f^{2}(\cdot, z)\right\rangle}, \quad \bar{r}^{\star}(z):=r_{\infty}-\frac{\langle\lambda(\cdot, z) f(\cdot, z)\rangle}{a} .
$$

Hence, we have that

$$
P_{0}(t, x, z)=\mathcal{B}_{V}\left(t, x ; T, \bar{\sigma}(z), \bar{r}^{\star}(z)\right),
$$

where $\mathcal{B}_{V}$ was defined in (17). This is the constant volatility Vasicek price evaluated at effective parameters $\bar{\sigma}(z)$ and $\bar{r}^{\star}(z)$, which result from averaging with respect to the fast variable, and from "freezing" the slow factor at its current level $z$. The explicit form of $P_{0}$ is given by

$$
P_{0}(t, x, z)=A(\tau, z) e^{-B(\tau) x},
$$

where $\tau=T-t$ is the time to maturity, and the expressions for $A(\tau, z)$ and $B(\tau)$ are now given by:

$$
\begin{aligned}
A(\tau, z) & =e^{-R_{\infty}(z) \tau+R_{\infty}(z) \frac{1}{a}\left(1-e^{-a \tau}\right)-\frac{\bar{\sigma}^{2}(z)}{4 a^{3}}\left(1-e^{-a \tau}\right)^{2}}, \\
B(\tau) & =\frac{1}{a}\left(1-e^{-a \tau}\right),
\end{aligned}
$$

with

$$
R_{\infty}(z)=\bar{r}^{\star}(z)-\frac{\bar{\sigma}^{2}(z)}{2 a^{2}}
$$

Define now $\widetilde{P}_{1,0}=\sqrt{\epsilon} P_{1,0}$, and the operator

$$
\mathcal{A}:=\sqrt{\epsilon}\left\langle\mathcal{L}_{1} \mathcal{L}_{0}^{-1}\left(\mathcal{L}_{2}-\left\langle\mathcal{L}_{2}\right\rangle\right)\right\rangle .
$$

The $\mathcal{O}(\sqrt{\epsilon})$ terms give that $\widetilde{P}_{1,0}$ is determined by the problem:

$$
\left\{\begin{aligned}
\left\langle\mathcal{L}_{2}\right\rangle \widetilde{P}_{1,0} & =\mathcal{A} P_{0} \\
\widetilde{P}_{1,0}(T, x, z) & =0
\end{aligned}\right.
$$

In order to obtain an expression for the operator $\mathcal{A}$ we introduce $\phi(y, z)$ and $\psi(y, z)$, solutions of the following Poisson equations with respect to $y$ :

$$
\begin{aligned}
& \mathcal{L}_{0} \phi(y, z)=f^{2}(y, z)-\bar{\sigma}^{2}(z) \\
& \mathcal{L}_{0} \psi(y, z)=\lambda(y, z) f(y, z)-\langle\lambda(\cdot, z) f(\cdot, z)\rangle .
\end{aligned}
$$

Both $\phi$ and $\psi$ are defined up to an additive function that does not depend on $y$, and that will not affect $\mathcal{A}$ since the operator $\mathcal{L}_{1}$ (which is included in $\mathcal{A}$ ) takes derivatives with respect to $y$. We then have

$$
\mathcal{L}_{0}^{-1}\left(\mathcal{L}_{2}-\left\langle\mathcal{L}_{2}\right\rangle\right)=\frac{1}{2} \phi(y, z) \frac{\partial^{2}}{\partial x^{2}}-\psi(y, z) \frac{\partial}{\partial x},
$$


and therefore

$$
\begin{array}{r}
\mathcal{L}_{1} \mathcal{L}_{0}^{-1}\left(\mathcal{L}_{2}-\left\langle\mathcal{L}_{2}\right\rangle\right)=\nu \sqrt{2}\left\{\rho_{1} f(y, z) \frac{\partial^{2}}{\partial x \partial y}\left(\frac{1}{2} \phi \frac{\partial^{2}}{\partial x^{2}}-\psi \frac{\partial}{\partial x}\right)\right. \\
\left.-\Lambda \frac{\partial}{\partial y}\left(\frac{1}{2} \phi \frac{\partial^{2}}{\partial x^{2}}-\psi \frac{\partial}{\partial x}\right)\right\} .
\end{array}
$$

Expanding, and using the fact that $P_{0}(t, x, z)$ does not depend on $y$, we have that

$$
\begin{aligned}
\mathcal{A} P_{0}= & \frac{\nu \sqrt{\epsilon}}{\sqrt{2}} \rho_{1}\left\langle f(\cdot, z) \phi_{y}(\cdot, z)\right\rangle \frac{\partial^{3} P_{0}}{\partial x^{3}} \\
& -\frac{\nu \sqrt{\epsilon}}{\sqrt{2}}\left(\left\langle\Lambda(\cdot, z) \phi_{y}(\cdot, z)\right\rangle+2 \rho_{1}\left\langle f(\cdot, z) \psi_{y}(\cdot, z)\right\rangle\right) \frac{\partial^{2} P_{0}}{\partial x^{2}} \\
& +\nu \sqrt{2 \epsilon}\left\langle\Lambda(\cdot, z) \psi_{y}(\cdot, z)\right\rangle \frac{\partial P_{0}}{\partial x},
\end{aligned}
$$

where $\phi_{y}$ represents the partial derivative of $\phi$ with respect to $y$, and the analogous convention holds for $\psi_{y}$. If we now let

$$
\begin{aligned}
V_{1}^{\epsilon}(z) & :=\nu \sqrt{2 \epsilon}\left\langle\Lambda \psi_{y}\right\rangle, \\
V_{2}^{\epsilon}(z) & :=-\frac{\nu \sqrt{\epsilon}}{\sqrt{2}}\left(\left\langle\Lambda \phi_{y}\right\rangle+2 \rho_{1}\left\langle f \psi_{y}\right\rangle\right), \\
V_{3}^{\epsilon}(z) & :=\frac{\nu \sqrt{\epsilon}}{\sqrt{2}} \rho_{1}\left\langle f \phi_{y}\right\rangle,
\end{aligned}
$$

then we can write

$$
\mathcal{A}=V_{1}^{\epsilon} \frac{\partial}{\partial x}+V_{2}^{\epsilon} \frac{\partial^{2}}{\partial x^{2}}+V_{3}^{\epsilon} \frac{\partial^{3}}{\partial x^{3}},
$$

The problem for $\widetilde{P}_{1,0}$ then becomes

$$
\left\{\begin{aligned}
\left\langle\mathcal{L}_{2}\right\rangle \widetilde{P}_{1,0} & =V_{1}^{\epsilon} \frac{\partial P_{0}}{\partial x}+V_{2}^{\epsilon} \frac{\partial^{2} P_{0}}{\partial x^{2}}+V_{3}^{\epsilon} \frac{\partial^{3} P_{0}}{\partial x^{3}} \\
\widetilde{P}_{1,0}(T, x, z) & =0 .
\end{aligned}\right.
$$

The problem for $\widetilde{P}_{1,0}$ can easily be solved explicitly, to give a representation in the form:

$$
\widetilde{P}_{1,0}=D^{\epsilon}(\tau, z) A(\tau, z) e^{-B(\tau) x},
$$

see $[4,6]$. However, before we give the explicit form we shall in Section 6 carry out a group parameter reduction that will simplify the representation of the solution. 


\subsection{The Slow Scale Correction}

Let us now expand $P_{1}^{\epsilon}$ (the second term on the expansion (44)) in terms of the small parameter $\epsilon$,

$$
P_{1}^{\epsilon}=P_{0,1}+\sqrt{\epsilon} P_{1,1}+\epsilon P_{2,1}+\epsilon^{3 / 2} P_{3,1}+\cdots,
$$

and substitute this expression in the terminal value problem that defines $P_{1}^{\epsilon}$ (equation (47)). The leading order term gives that $P_{0,1}$ does not depend on $y$, that is, $P_{0,1}=P_{0,1}(t, x, z)$.

Define $\widetilde{P}_{0,1}=\sqrt{\delta} P_{0,1}$ and $\widetilde{\mathcal{M}}_{1}=\sqrt{\delta} \mathcal{M}_{1}$. From the $\mathcal{O}(1)$ terms we obtain that the problem that determines $\widetilde{P}_{0,1}$ is

$$
\left\{\begin{aligned}
\left\langle\mathcal{L}_{2}\right\rangle \widetilde{P}_{0,1} & =-\left\langle\widetilde{\mathcal{M}}_{1}\right\rangle P_{0} \\
\widetilde{P}_{0,1}(T, x, z) & =0
\end{aligned}\right.
$$

where we recall that $P_{0}=A(\tau, z) e^{-B(\tau) x}$. Let

$$
\begin{aligned}
V_{0}^{\delta}(z) & =-\sqrt{\delta} g(z)\langle\Gamma(\cdot, z)\rangle \\
V_{1}^{\delta}(z) & =\sqrt{\delta} \rho_{2} g(z)\langle f(\cdot, z)\rangle
\end{aligned}
$$

and recall that $\mathcal{M}_{1}$ is given in (41). Then we can write $\left\langle\widetilde{\mathcal{M}}_{1}\right\rangle$, as

$$
\left\langle\widetilde{\mathcal{M}}_{1}\right\rangle=V_{0}^{\delta} \frac{\partial}{\partial z}+V_{1}^{\delta} \frac{\partial^{2}}{\partial x \partial z}
$$

Again, the problem for $\widetilde{P}_{0,1}$ can easily be solved explicitly, to give a representation in the form:

$$
\widetilde{P}_{0,1}=D^{\delta}(\tau, z) A(\tau, z) e^{-B(\tau) x},
$$

see [6]. However, in Section 6 we carry out a group parameter reduction that will simplify the representation of this solution. Before we go into the details of the group parameter transformation we present in the next section a numerical illustration of some typical corrections to the bond and yield that derives from our multiscale model.

\subsection{The Bond Price Approximation}

In We define the bond price approximation as

$$
\widetilde{P}^{\epsilon, \delta}:=P_{0}+\sqrt{\epsilon} P_{1,0}+\sqrt{\delta} P_{0,1}=P_{0}+\widetilde{P}_{1,0}+\widetilde{P}_{0,1}
$$

From (67), (72) and (53) it follows that we can write the approximation explicitly as

$$
\widetilde{P}^{\epsilon, \delta}=\left(1+D^{\epsilon}+D^{\delta}\right) A e^{-B x},
$$


where $D^{\epsilon}$ and $D^{\delta}$ are $\mathcal{O}(\sqrt{\epsilon})$ and $\mathcal{O}(\sqrt{\delta})$ respectively and $A$ and $B$ are defined in (54) and (55). The corresponding approximation to the yield curve, $\widetilde{R}^{\epsilon, \delta}=$ $-\frac{1}{\tau} \log \widetilde{P}^{\epsilon, \delta}$, is given by

$$
\widetilde{R}^{\epsilon, \delta}=R_{0}-\frac{1}{\tau} \log \left(1+D^{\epsilon}+D^{\delta}\right) \approx R_{0}-\frac{1}{\tau}\left(D^{\epsilon}+D^{\delta}\right),
$$

where $R_{0}=-\frac{1}{\tau} \log P_{0}$ is the yield corresponding to the constant volatility price $P_{0}$.

The influence of the corrections $D^{\epsilon}$ and $D^{\delta}$ will affect the shape of the yield curve, so we expect a richer variety of shapes.

In the following figures we use the values of the parameters $a=1, \bar{\sigma}=0.1$, $\bar{r}^{\star}=0.1$ and the initial value $x=0.07$ and show results corresponding to the approximation derived above. If we assume that only the fast scale process $Y_{t}$ has influence on $\sigma_{t}$ (i.e., we assume $\delta=0$ ), we obtain the graphics in Figure 3 , where the prices and yields are computed for $\epsilon=0.001$. The yield curve is increasing for very short maturities, it becomes decreasing for medium range maturities (from 1.5 years to 9 years) and then it becomes slowly increasing again.

In Figure 4 we assume that only the slow scale process affects the volatility (i.e., we take $\epsilon=0$ ). The yield curve is increasing up to 6 years and it decreases for longer maturities. Figure 5 shows the case when the volatility is driven by both processes, $Y_{t}$ and $Z_{t}$. As one would expect, it seems that the slow scale seems to have a greater impact on the long range, while the fast scale seems to affect the medium maturity yields. In Figure 6 we can see that the larger the value of $\delta$, the more pronounced is the influence of the slow scale volatility.

\section{Group Parameter Reduction}

The leading order bond price, $P_{0}$, depends on the parameters $a, \bar{\sigma}(z)$ and $\bar{r}^{\star}(z)$, which are those that define the operator $\mathcal{L}_{V}\left(\bar{\sigma}, \bar{r}^{\star}\right)$ (see (51)). The first order corrections, $\widetilde{P}_{1,0}$ and $\widetilde{P}_{0,1}$, depend in particular also on the $z$-dependent group parameters

$$
V_{1}^{\epsilon}, V_{2}^{\epsilon}, V_{3}^{\epsilon}, V_{0}^{\delta}, V_{1}^{\delta}
$$

If we define the price correction

$$
P_{c}^{\epsilon, \delta}:=\sqrt{\epsilon} P_{1,0}+\sqrt{\delta} P_{0,1},
$$

then the problem characterizing $P_{c}^{\epsilon, \delta}$ can be written as

$$
\left\{\begin{aligned}
\mathcal{L}_{V}\left(\bar{\sigma}, \bar{r}^{\star}\right) P_{c}^{\epsilon, \delta} & =-\mathcal{H}_{V}^{\epsilon, \delta} P_{0} \\
P_{c}^{\epsilon, \delta}(T, x, z ; T) & =0
\end{aligned}\right.
$$



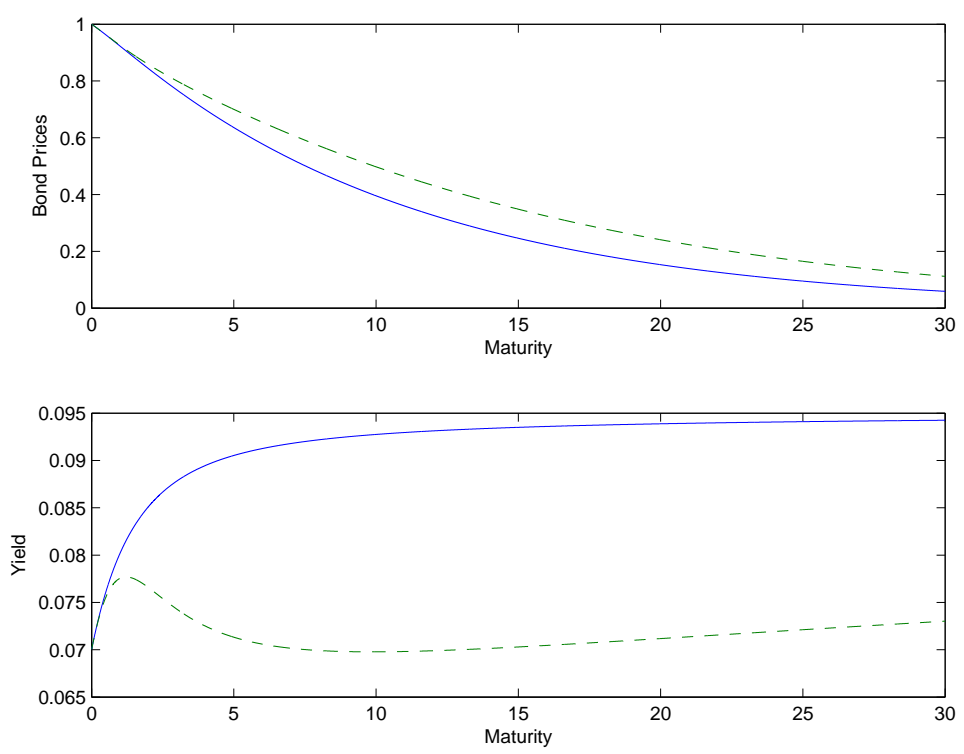

Figure 3: Bond price and yield curves with constant volatility (thin line), and fastscale stochastic volatility (dashed line). For this example, $\epsilon=0.001$.
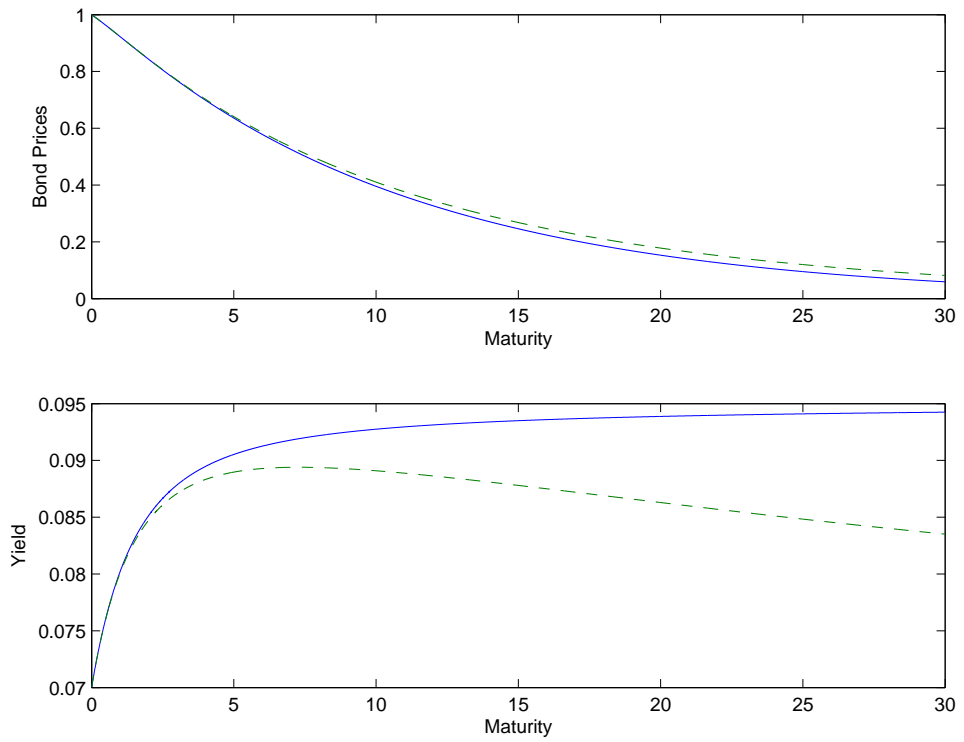

Figure 4: Bond price and yield curves with constant volatility (thin line), and slowscale stochastic volatility (dashed line). For this example, $\delta=0.01$. 

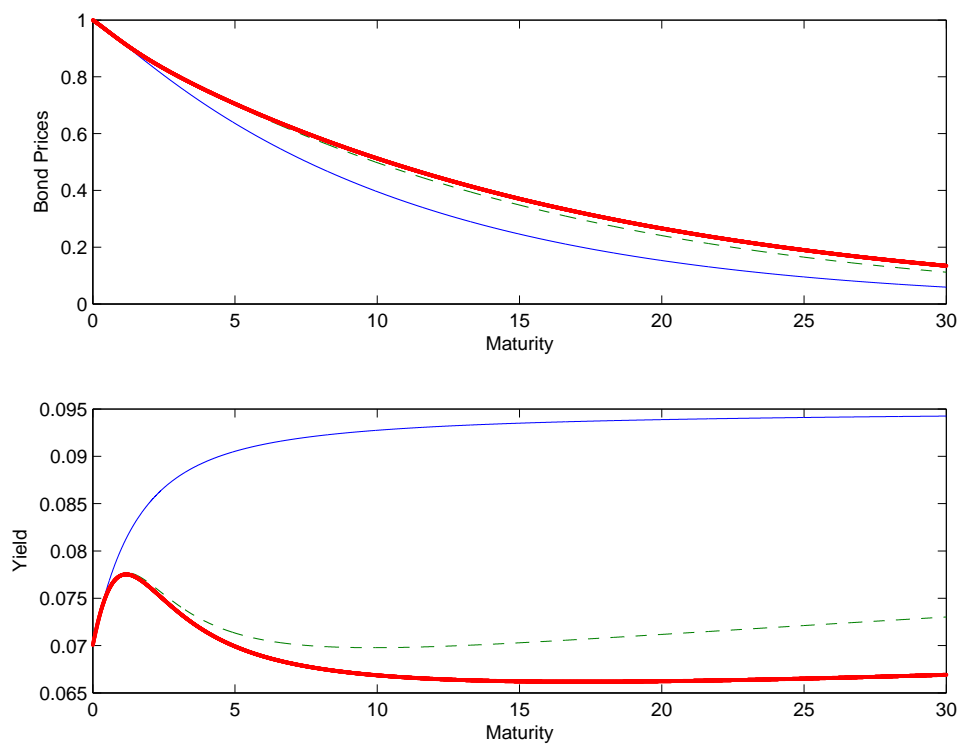

Figure 5: Bond price and yield curves with constant volatility (thin line), fast-scale stochastic volatility (dashed line) and two-factor stochastic volatility (dotted line). For this example, $\epsilon=0.001, \delta=0.01$.
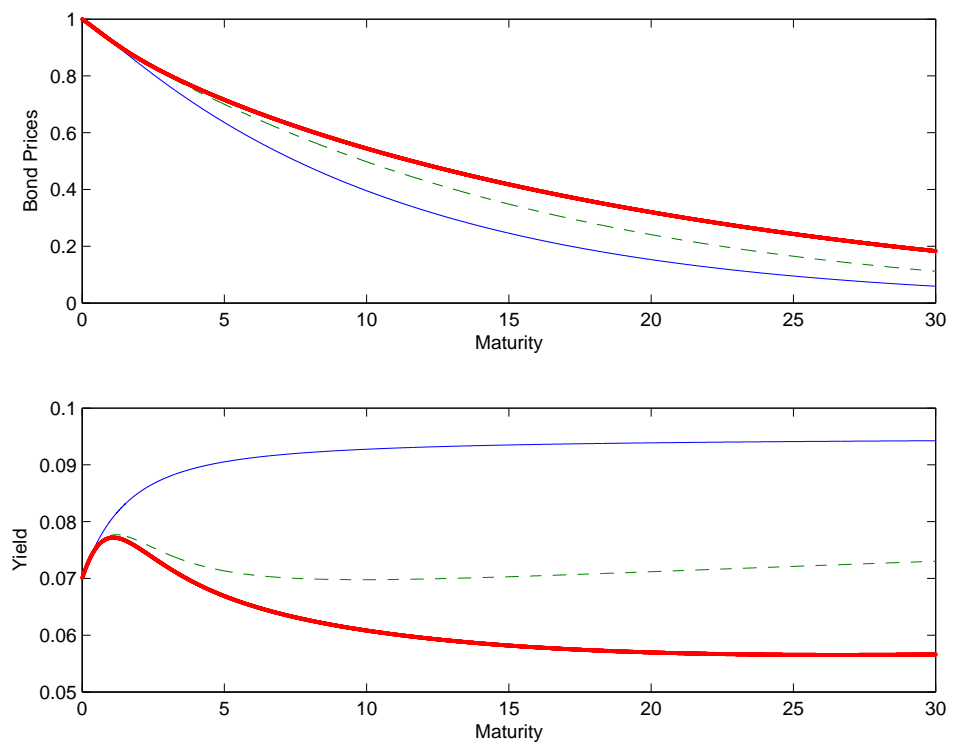

Figure 6: Bond prices and yields as in Figure 5, when $\epsilon=0.001, \delta=0.1$. 
where in the source term we have used the notation

$$
\mathcal{H}_{V}^{\epsilon, \delta}=\sum_{k=1}^{3} V_{k}^{\epsilon} \frac{\partial^{k}}{\partial x^{k}}+\left(V_{0}^{\delta} \frac{\partial}{\partial z}+V_{1}^{\delta} \frac{\partial^{2}}{\partial z \partial x}\right) .
$$

Note that the price approximation does not depend on $y$, and that $z$ is a fixed parameter (obtained by "freezing" the slow factor at its current level). In this section we discuss how to effectively reduce the number of degrees of freedom.

If we now define

$$
\begin{aligned}
m_{1} & :=\frac{\partial R_{\infty}(z)}{\partial z} \\
m_{2} & :=\frac{\partial\left(\bar{\sigma}^{2}(z) /(4 a)\right)}{\partial z}
\end{aligned}
$$

by making use of (53) and (54), we can write the source operator in terms of the "Greeks" as

$$
-\mathcal{H}_{V}^{\epsilon, \delta} P_{0}=-\left\{U_{1} \frac{\partial}{\partial x}+U_{2} \frac{\partial^{2}}{\partial x^{2}}+U_{3} \frac{\partial^{3}}{\partial x^{3}}-\tau m_{1}\left(V_{0}^{\delta}+V_{1}^{\delta} \frac{\partial}{\partial x}\right)\right\} P_{0},
$$

with

$$
U_{1}=V_{1}^{\epsilon}-m_{1} V_{0}^{\delta}, \quad U_{2}=V_{2}^{\epsilon}-m_{2} V_{0}^{\delta}-m_{1} V_{1}^{\delta}, \quad U_{3}=V_{3}^{\epsilon}-m_{2} V_{1}^{\delta} .
$$

If we now define

$$
\begin{aligned}
\sigma^{\star}(z) & =\sqrt{\bar{\sigma}^{2}(z)+2 U_{2}(z)}, \\
r^{\star \star}(z) & =r^{\star}(z)+\frac{U_{1}(z)}{a},
\end{aligned}
$$

we have that

$$
\mathcal{L}_{V}\left(\sigma^{\star}, r^{\star \star}\right)=\mathcal{L}_{V}\left(\bar{\sigma}, \bar{r}^{\star}\right)+U_{2} \frac{\partial^{2}}{\partial x^{2}}+U_{1} \frac{\partial}{\partial x}
$$

where we have dropped the $z$ dependence. Therefore, using (50), (77) and the fact that $\widetilde{P}^{\epsilon, \delta}=P_{0}+P_{c}^{\epsilon, \delta}$, we get that

$$
\mathcal{L}_{V}\left(\sigma^{\star}, r^{\star \star}\right) \widetilde{P}^{\epsilon, \delta}=U_{1} \frac{\partial P_{c}^{\epsilon, \delta}}{\partial x}+U_{2} \frac{\partial^{2} P_{c}^{\epsilon, \delta}}{\partial x^{2}}-U_{3} \frac{\partial^{3} P_{0}}{\partial x^{3}}+\tau m_{1}\left(V_{0}^{\delta}+V_{1}^{\delta} \frac{\partial}{\partial x}\right) P_{0},
$$

where the source is very similar to (79), except for the first two terms. Note that the source terms

$$
U_{1} \frac{\partial P_{c}^{\epsilon, \delta}}{\partial x} \quad \text { and } \quad U_{2} \frac{\partial^{2} P_{c}^{\epsilon, \delta}}{\partial x^{2}}
$$


are of order $\mathcal{O}(\epsilon+\delta)$, since $V_{1}^{\epsilon}, V_{2}^{\epsilon}$ and $\widetilde{P}_{1,0}$ are all of order $\mathcal{O}(\sqrt{\epsilon})$, and $V_{0}^{\delta}$, $V_{1}^{\delta}$ and $\widetilde{P}_{0,1}$ are all of order $\mathcal{O}(\sqrt{\delta})$. Thus, the first two terms of the source are negligible compared to the other source terms, and therefore the corrected price, $P_{0}+P_{c}^{\epsilon, \delta}$, has the same order of accuracy as $P_{0}^{\star}+P_{c}^{\star}$, where $P_{0}^{\star}$ satisfies

$$
\left\{\begin{array}{r}
\mathcal{L}_{V}\left(\sigma^{\star}, r^{\star \star}\right) P_{0}^{\star}=0 \\
P_{0}^{\star}(T, x ; T)=1
\end{array}\right.
$$

and $P_{c}^{\star}$ satisfies

$$
\left\{\begin{aligned}
\mathcal{L}_{V}\left(\sigma^{\star}, r^{\star \star}\right) P_{c}^{\star} & =-\mathcal{H}_{V}^{\star} P_{0}^{\star} \\
P_{c}^{\star}(T, x ; T) & =0
\end{aligned}\right.
$$

with the new source operator

$$
\mathcal{H}_{V}^{\star}=U_{3} \frac{\partial^{3}}{\partial x^{3}}-\tau\left(W_{0}+W_{1} \frac{\partial}{\partial x}\right),
$$

where we defined

$$
W_{0}=m_{1} V_{0}^{\delta}, \quad W_{1}=m_{1} V_{0}^{\delta} .
$$

Note that the set of parameters that are to be calibrated is thus reduced to, first, the $\mathcal{O}(1)$ parameters

$$
a, \quad r^{\star \star}, \quad \sigma^{\star},
$$

in addition, we have the small parameters

$$
U_{3}, \quad W_{0}, \quad W_{1},
$$

that are $\mathcal{O}(\sqrt{\epsilon}+\sqrt{\delta})$.

\subsection{Yield Correction}

From (67) and (72) we know that the first order correction to the bond price can be written as $P_{c}^{\epsilon, \delta}=\left(D^{\epsilon}+D^{\delta}\right) P_{0}$. Because $P_{c}^{\star}$ has the same order of accuracy as $P_{c}^{\epsilon, \delta}$, we may write

$$
P_{c}^{\star}=D^{\star} P_{0}^{\star},
$$

so that the corrected yield takes the form

$$
\widetilde{R}^{\epsilon, \delta}=R_{0}^{\star}-\frac{1}{\tau} \log \left(1+D^{\star}\right) \approx R_{0}^{\star}-\frac{1}{\tau} D^{\star} .
$$

Here $R_{0}^{\star}=-\frac{1}{\tau} \log P_{0}^{\star}$ is the yield corresponding to the constant volatility bond price $P_{0}^{\star}$ evaluated at the effective short rate and volatility $\left(r^{\star \star}, \sigma^{\star}\right)$, and

$$
D^{\star}(\tau)=U_{3} g_{1}(\tau)+W_{0} g_{2}(\tau)+W_{1} g_{3}(\tau),
$$


with

$$
\begin{aligned}
g_{1}(\tau) & =\frac{B(\tau)-\tau}{a^{3}}+\frac{B^{2}(\tau)}{2 a^{2}}+\frac{B^{3}(\tau)}{3 a}, \\
g_{2}(\tau) & =-\tau^{2} / 2 \\
g_{3}(\tau) & =\frac{\tau}{a^{2}}+\frac{\tau^{2}}{2 a}-B(\tau)\left(\frac{\tau}{a}+\frac{1}{a^{2}}\right) .
\end{aligned}
$$

Note that in this parameterization the structure of the yield curve correction is not affected by the fast factor as long as the slow is present, only the interpretation of the parameters does. Observe also that only the temporal aspect of the yield curve is affected by the stochastic volatility modulation. The spatial part is still determined by the modulation $x B(\tau) / \tau$ in the expression for the leading yield surface

$$
R_{0}(t, \tau ; x, z)=R_{\infty}^{\star}+\left(x-R_{\infty}^{\star}\right) \frac{B(\tau)}{\tau}+\frac{\left(\sigma^{\star}\right)^{2}}{4 a} \frac{B^{2}(\tau)}{\tau}
$$

where we defined

$$
R_{\infty}^{\star}=r^{\star \star}(z)-\frac{\left(\sigma^{\star}\right)^{2}(z)}{2 a^{2}} .
$$

Observe that in order to calibrate the parameters $c_{i}, i=1,2,3$, we would need to regress the observed yield corrections relative to the constant volatility model against the yield curve terms factors $g_{i}, i=1,2,3$. We comment on the calibration in more detail in the next section.

\section{Calibration of the Model}

In this section we discuss one way of calibrating the model to market data. Assume we for various $t$ and $T$ can observe the yield $R(t, T ; x)$, the market yield at time $t$ of a bond with maturity $T$ and current short rate level $x$ and time to maturity $\tau=T-t$. To emphasize that it is an observed market yield we will write $R^{o b s}(t, T)$. We then seek to estimate

$$
a, \quad r^{\star \star}, \quad \sigma^{\star}, \quad U_{3}, \quad W_{0}, \quad W_{1}
$$

so that for the set of observed yields we have

$$
R^{o b s}(t, T ; x) \approx R_{0}^{\star}\left(\tau, a, r^{\star \star}, \sigma^{\star} ; x\right)-\left(U_{3} g_{1}(\tau)+W_{0} g_{2}(\tau)+W_{1} g_{3}(\tau)\right) / \tau,
$$

where $R_{0}^{\star}$ corresponds to the constant parameter Vasicek yield as given in (25), but evaluated at the corrected parameters. This can for instance be accomplished by first fitting $R_{0}^{\star}$ to the data by estimating the $\mathcal{O}(1)$ parameters $a, r^{\star \star}, \sigma^{\star}$ via a least squares procedure to get apriori estimates of their leading value. The multiscale correction will affect the "wings" of the yield term structure relatively strongest, corresponding to small and large maturity. Thus, in 
a second step we include the correction terms and estimate also the small correction parameters in addition to an updated estimate of the parameters $r^{\star \star}, \sigma^{\star}$. Note that when exploiting the apriori parameter estimates this second step becomes, in view of the form (25), actually a linear least squares problem relative to a set of term structure factors defined in terms of the apriori estimates.

We conclude that the asymptotic framework has provided us with a robust approach to parameterize the yield term structure, moreover, the parameters of the fitted term structure can be used in the pricing of related, potentially less liquid, contracts.

\section{Connection to Defaultable Bonds}

We consider a particular obligor corresponding to an underlying name. The event that the obligor defaults is modeled in terms of the first arrival of a Poisson process with stochastic intensity or hazard rate $\lambda^{(1)}$. Conditioned on the path of the hazard rate the probability that the obligor has survived till time $T$ is thus $\exp \left(-\int_{0}^{T} \lambda_{s}^{(1)} d s\right)$. The probability of survival till time $T$ is then under the doubly stochastic framework

$$
E^{\mathcal{Q}}\left[e^{-\int_{0}^{T} \lambda_{s}^{(1)} d s}\right],
$$

with the expectation taken with respect to the risk neutral of pricing measure so that this corresponds to the bond price expression (3). In the Vasicek setup we model the intensity by a Vasicek process so that

$$
\lambda_{t}^{(1)}=X_{t}^{(1)},
$$

is an Ornstein-Uhlenbeck processes:

$$
d X_{t}^{(1)}=a_{1}\left(X_{1}^{\star}-X_{t}^{(1)}\right) d t+\sigma_{1} d W_{t}^{(1)} .
$$

Thus, we can re-interpret all our results on multiscale stochastic volatility bond prices as results on the survival probability in the context of a defaultable bond in the case where the underlying hazard rate process is modelled in terms of a multiscale stochastic volatility Vasicek process, see also [10, 14]. In the multiname case correlations in between name is essential and there is an important "gearing" effect of stochastic volatility impact in terms of the size of the name portfolio regarding its joint survival probability, see $[5,15]$.

\section{References}

[1] T. Andersen and J. Lund. Estimating Continuous-time Stochastic Volatility Models of the Short-term Interest Rate. Journal of Econometrics, 77, 343-377, 1997. 
[2] T. Bjork, Arbitrage Theory in Continuous Time Oxford University Press, 1998.

[3] R. Brennen and R. Harjes and K. Kroner. Another Look at Alternative Models of the Short-term Interest Rate. Journal of Financial and Quantitative Analysis, 1, 85-107, 1996.

[4] P. Cotton, J.P. Fouque, G. Papanicolaou and R. Sircar. Stochastic Volatility Corrections for Interest Rates Models, Mathematical Finance, 14, 173200, 2004 .

[5] P. Cotton, J.P. Fouque, R. Sircar and K. Solna. Multiname and Multiscale Default Modeling, Preprint.

[6] R. De Santiago. Interest Rate Derivatives and Value-at-Risk with Multiscale Stochastic Volatility. Ph.D. Thesis. University of California-Irvine, 2007.

[7] J.P. Fouque, G. Papanicolaou, and K.R. Sircar. Derivatives in Financial Markets with Stochastic Volatility. Cambridge University Press, 2000.

[8] J.P. Fouque, G. Papanicolaou, R. Sircar and K. Sølna. Singular Perturbations in Option Pricing. SIAM Journal on Applied Mathematics 63(5), 1648-1665 2003.

[9] J.P. Fouque, G. Papanicolaou, K.R. Sircar and K. Solna. Multiscale Stochastic Volatility Asymptotics. SIAM Journal Multiscale Modeling and Simulation, 2, 22-42, 2003.

[10] J.P. Fouque, R. Sircar and K. Sølna. Stochastic Volatility Effects on Defaultable Bonds, Applied Mathematical Finance, 13, 215-244, 2006.

[11] J.-P. Fouque, B.C. Wignall and X. Zhou. Modeling Correlated Defaults: First Passage Model under Stochastic Volatility, Submitted 2006.

[12] J.-P. Fouque and X. Zhou. Perturbed Gaussian Copula, this volume.

[13] I. Karatzas and S. Shreve. Methods of Mathematical Finance, SpringerVerlag. 1998.

[14] E. Papageorgiou and R. Sircar. Multiscale Intensity Models and Name Grouping for Valuation of Multi-name Credit Derivatives, submitted.

[15] E. Papageorgiou and R. Sircar. Multiscale Intensity Models for Single Name Credit Derivatives, to appear in Applied Mathematical Finance. 